\title{
Dynamics of Anisotropically Supported Rotors
}

\author{
AGNES MUSZYNSKA, Ph.D.*, CHARLES T. HATCH and DONALD E. BENTLY \\ Bently Rotor Dynamics Research Corporation, P.O. Box 2529, Minden, Nevada 89423
}

(Received 15 May 1996; In final form 14 June 1996)

\begin{abstract}
The paper discusses dynamic effects occurring in machinery rotors supported in bearings and pedestals with laterally different characteristics. In the considered rotor model the anisotropy of radial stiffness and tangential ("cross") stiffness components are included. Within certain ranges of the rotative speed the support anisotropy leads to the specific, excited-by-unbalance rotor lateral synchronous vibrations in a form of backward (reverse) precession. In addition, one section of the rotor may precess backward, while the other section simultaneously precesses forward. Experimental results illustrate this phenomenon. The analytical model of the system is based on multimode modal approach. It is also shown in this paper that greatly enhanced information for machine malfunction diagnostics can be obtained by simulated rotation of the $X Y$ transducer system observing rotor lateral vibration. This simulated rotation can be accomplished by the machine diagnostic data acquistion and processing system. The data processing also includes extraction of forward and backward components of elliptical orbits filtered to one frequency, and the filtered orbit major axis magnitude and its angular orientation.

Numerical examples, field data, and experimental results performed on a rotor rig illustrate applications.
\end{abstract}

Keywords: Rotating machine dynamics, rotor support anisotropy, rotor forward and backward precession, vibration diagnostics of machinery

\section{INTRODUCTION}

Most rotating machine support structures are characterized by lateral anisotropy. The anisotropy of the rotor system can originate in bearing support pedestals, foundations, and/or asymmetric piping attachments to the machine casing. It can also originate in fluid-lubricated bearings or seals, and process flow asymmetries. The anisotropy can affect mass, damping, and stiffness matrices. In effect, the rotor mode character- istics become anisotropic in two lateral orthogonal directions. This results in closely spaced, coupled "pairs" of rotor lateral modes revealed, for instance, in rotor $1 \times$ (synchronous) response polar and Bode plots as "split resonances." Due to the anisotropy, the $1 \times$ orbits excited in response to simple unbalance are elliptical with various degrees of ellipticity. It has been known that in certain rotative speed regions the rotor unbalance response orbits are backward (reverse).

*Corresponding author. Tel.: (702) 782-3611. Fax: (702) 782-9236. E-mail: agnes@brdrc.com. 
This classical effect is discussed in papers and books on rotordynamics, such as Gunter et al [1993], Vance [1993], and Handbook of Rotordynamics [1993]. With a specific unbalance distribution along the rotor axis, it may also happen that a portion of the rotor would precess forward, while another one precesses backward. This fact, briefly mentioned by Vance [1993], is discussed in this paper.

Vibration monitoring systems installed on rotating machines include a number of pairs of rotor displacement measuring transducers mounted at or nearby bearings in the orthogonal $X Y$ configuration. A specific transducer angular orientation seldom coincides with the support structure major or minor axis of stiffness anisotropy. In addition, these axes are usually nonorthogonal. Independently from the transducer lateral location, following the "oscilloscope convention," the vibrational information from both $X Y$ transducers is used to correctly recreate the rotor orbits, the magnified images of the rotor centerline motion. The transducer information is also used to obtain rotor filtered single frequency response vectors, such as $1 \times$ or $2 \times$ in the Bode and polar plot formats. For the purpose of these plots, the information from only one lateral transducer is required, thus the Bode and polar plot display data is characteristic for the specific transducer location. The anisotropy affects the response vectors, which observed from a different angular location, would be different. The questions arise about how to properly identify the unbalance ("heavy spot") angular location, especially at low speed, and how to evaluate the Synchronous Amplification Factors for anisotropic rotors. The fact is that the response phase and amplitude magnitudes vary significantly with observation angle.

The problems mentioned above are discussed in this paper using, as an example, a mathematical model of a two-mode rotor, based on the multimode modal approach, discussed by Muszynska [1994]. This model includes stiffness and tangential force anisotropy.

\section{MATHEMATICAL MODEL OF A TWO-MODE ANISOTROPIC ROTOR}

Consider two modes of a laterally symmetric rotor supported in anisotropic susceptible pedestals. The rotor model which includes anisotropic tangential force is as follows:

$$
\begin{gathered}
M \ddot{x}+D_{s} \dot{x}+K_{x} x+D\left(\dot{x}+\lambda_{x} \Omega y\right)=F \cos (\omega t+\delta) \\
M \ddot{y}+D_{s} \dot{y}+K_{y} y+D\left(\dot{y}-\lambda_{y} \Omega x\right)=F \sin (\omega t+\delta) \\
\cdot=\mathrm{d} / \mathrm{d} t
\end{gathered}
$$

where $x(t), y(t)$ are rotor orthogonal lateral deflections, $M, D_{s}$ are rotor modal mass and damping respectively, $D$ is surrounding fluid radial damping, $\lambda_{.}$, $\lambda_{y}$ are fluid circumferential average velocity ratios [Muszynska, 1994], $\Omega$ is rotative speed, $K_{x}, K_{y}$ are rotor/supporting structure stiffnesses in $x$ and $y$ directions. For positive $\lambda_{x}$ and $\lambda_{y}$ the expressions $D \lambda_{x} \Omega y$ and $-D \lambda_{y} \Omega x$ represent nonsymmetric components of a forward (acting in the direction of rotation) tangential force. This force is due to circumferential flow of the rotor surrounding fluid (process and/or lubricating fluid). As discussed, for instance, in the Handbook of Rotordynamics [1993], the tangential force may also originate from other sources. The parameters $F, \omega$ and $\delta$ denote external exciting, nonsynchronously rotating force amplitude, frequency, and angular orientation, respectively.

Muszynska [1989] showed that equations (1) can be solved analytically. There. exist two cases: (a) weak coupling, and (b) strong coupling, for which the eigenvalues and modal functions are slightly different. In case (b) instability may occur. The results are summarized in Table I.

The solution of Eqs. (1) forced by the external nonsynchronous rotating force is as follows:

$$
x=A_{x} \cos \left(\omega t+\alpha_{x}\right), \quad y=A_{y} \cos \left(\omega t+\alpha_{y}\right)
$$

where the response amplitudes $A_{x}, A_{y}$ and phases $\alpha_{x}$, $\alpha_{y}$ are as follows $\left(A_{x} \mathrm{e}^{j \alpha_{1}}, A_{y} \mathrm{e}^{j \alpha_{v}}\right.$ are called response vectors):

$$
\begin{gathered}
A_{x}=\frac{F}{\Delta} \sqrt{L_{y}^{2}+H_{x}^{2}}, A_{y}=\frac{F}{\Delta} \sqrt{L_{x}^{2}+H_{y}^{2}}, \\
L_{y}=K_{y}-M \omega^{2}, H_{y}=\left(D+D_{s}\right) \omega+D \lambda_{y} \Omega
\end{gathered}
$$


TABLE I Eigenvalue Solution for Eqs. (1)

\begin{tabular}{|c|c|c|}
\hline Case & $\begin{array}{l}\text { (a) Weak Coupling: } \\
4 D^{2} \Omega^{2} \lambda_{x} \lambda_{y} \leq\left(K_{x}-K_{y}\right)^{2}\end{array}$ & $\begin{array}{c}\text { (b) Strong Coupling: } \\
4 D^{2} \Omega^{2} \lambda_{x} \lambda_{y}>\left(K_{x}-K_{y}\right)^{2}\end{array}$ \\
\hline Eigenvalues $s_{i}, s_{i+2}, i=1,2$ & $-\epsilon \pm j \omega_{i}$ & $-\epsilon \pm\left[\mu+j(-1)^{i} \beta\right]$ \\
\hline Instability Threshold $\Omega_{\iota \prime}$ & $+\cdots-\cdot$ & $\frac{1}{\sqrt{\lambda_{x} \lambda_{y}}} \sqrt{\left(1+\frac{D_{s}}{D}\right)^{2} \frac{K_{x}+K_{y}}{2 M}+\left(\frac{K_{x}-K_{y}}{2 D}\right)^{2}}$ \\
\hline $\begin{array}{l}\text { Eigenfunctions } \\
\phi_{1} \text { for } s_{1} \& s_{3} \\
\phi_{2} \text { for } s_{2} \& s_{4}\end{array}$ & $\phi_{i}=\frac{K_{y}-K_{x}+(-1)^{i} 2 M \sqrt{G_{2}}}{2 D \lambda_{r} \Omega}$ & $\phi_{i}=\sqrt{\frac{\lambda_{y}}{\lambda_{x}}} e^{j \gamma(-1)^{i}}, \quad i=1,2$ \\
\hline Notation: $\epsilon=\left(D+D_{s}\right) / 2 M$ & 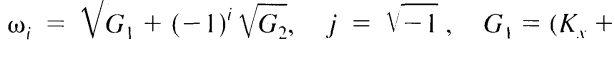 & $2 M-\epsilon^{2}, \quad G_{2}=\left[\left(K_{x}-K_{y}\right)^{2}-4 D^{2} \Omega^{2} \lambda_{x} \lambda_{y}\right] / 4 M^{2}$ \\
\hline$\mu=\sqrt{-G_{1}+\sqrt{G_{1}^{2}-G_{2}}} / \sqrt{2}$ & $\beta=\sqrt{G_{1}+\sqrt{G_{1}^{2}-G_{2}}} / \sqrt{2}, \quad \gamma=\arctan \frac{2 M \sqrt{-G_{2}}}{\left|K_{x}-K_{y}\right|}$ & \\
\hline
\end{tabular}

$$
\begin{gathered}
\Delta=\sqrt{L_{\Delta}^{2}+H_{\Delta}^{2}}, L_{\Delta}=L_{x} L_{y}-\left(D+D_{s}\right)^{2} \omega^{2} \\
+D^{2} \Omega^{2} \lambda_{x} \lambda_{y}, \alpha_{x}=\delta+\arctan \frac{H_{x}}{L_{y}}-\alpha, \\
H_{\Delta}=\omega\left(D+D_{s}\right)\left(K_{x}+K_{y}-2 M \omega^{2}\right), \\
\alpha_{y}=\delta+\arctan \frac{H_{y}}{L_{x}}-\alpha-90^{\circ}, \alpha=\arctan \frac{H_{\Delta}}{L_{\Delta}}
\end{gathered}
$$

In the particular case where the excitation is synchronous, resulting from the rotor unbalance, then $\omega$ $=\Omega$. The Bode and polar plots of the rotor synchronous and nonsynchronous responses for a set of numerical values are shown in Figures 1 and 2. Unbalance-type force excitation was assumed, thus $F=$ $m r \omega^{2}$. Response amplitudes were dimensionalized by multiplying them by $M / m r$, therefore, at infinite frequency the response amplitude converges to one. The Amplification Factor, defined as the ratio of amplitudes at resonance and at infinite frequency, can be read directly as the nondimensional amplitude value at resonance.

\section{VIBRATION DATA PROCESSING FOR MODE DECOUPLING: TRANSDUCER ROTATION SIMULATION}

In machine monitoring systems the displacement transducers observing the rotor are mounted in $X Y$ configuration which usually does not coincide with the major or minor rotor/support stiffness axis directions. The vibrational data obtained from the transducers most often indicate some level of the system anisotropy: $1 \times$ orbits are elliptical in a broad rotative speed range, $1 \times$ Bode and polar plots display "split resonances." An improvement for easier interpretation of such data can be achieved if the $1 \times$ response vectors obtained from $X$ and $Y$ transducers are post processed, in particular, rotated by an angle $\Theta$ (Fig. 3). The new orthogonal response vectors $A_{u} \mathrm{e}^{j \alpha_{u}}, A_{u} \mathrm{e}^{j \alpha_{u}}$ will have the following amplitudes and phases:

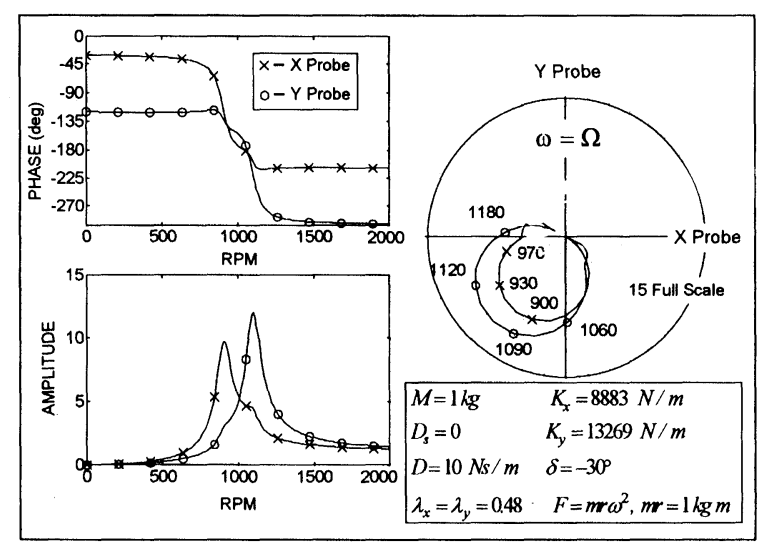

FIGURE 1 Synchronous $(1 \times)$ response vectors of the rotor $(\omega=$ $\Omega$ ) in Bode and polar plot format, calculated from Eqs. (1). The phase crossing in the range of 1070 to $1160 \mathrm{rpm}$ indicates backward orbiting. Data from the $Y$ probe on the polar plot is rotated by $90^{\circ}$ to coincide better with data from the $X$ probe (in case of isotropic rotor the polar circles are identical). 


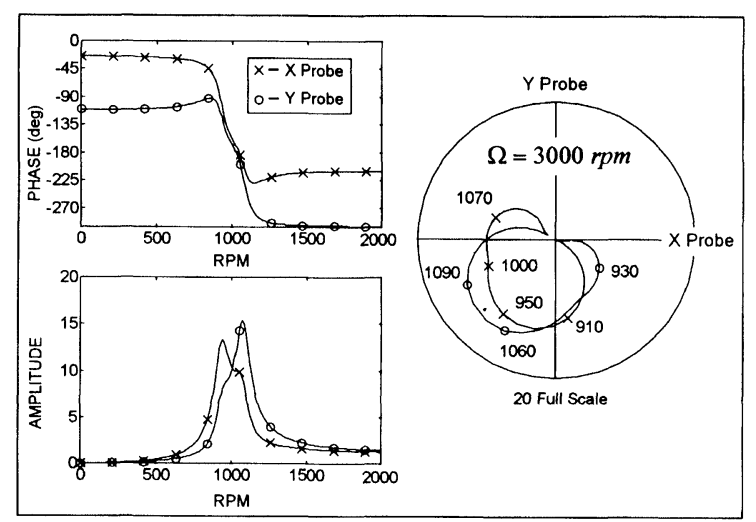

FIGURE 2 Nonsynchronous response vectors of the rotor for $\Omega$ $=3000 \mathrm{rpm}$ in Bode and polar plot format, calculated from Eqs. (1). Note a difference in amplitude in comparison with Fig. 1.

$$
A_{u}=
$$

$\sqrt{A_{x}^{2} \cos ^{2} \Theta+A_{y}^{2} \sin \Theta+A_{x} A_{y} \sin 2 \Theta \cos \left(\alpha_{x}-\alpha_{y}\right)}$,

$$
A_{11}=
$$

$\sqrt{A_{x}^{2} \sin ^{2} \Theta+A_{y}^{2} \cos ^{2} \Theta-\mathrm{A}_{\mathrm{x}} \mathrm{A}_{\mathrm{y}} \sin 2 \Theta \cos \left(\alpha_{x}-\alpha_{y}\right)}$,

$$
\alpha_{u}=\arctan \frac{A_{x} \sin \alpha_{x}+A_{y} \sin \alpha_{y} \tan \Theta}{A_{x} \cos \alpha_{x}+A_{y} \cos \alpha_{y} \tan \Theta},
$$$$
\alpha_{11}=\arctan \frac{A_{x} \sin \alpha_{x} \tan \Theta-A_{y} \sin \alpha_{y}}{A_{x} \cos \alpha_{x} \tan \Theta-A_{y} \cos \alpha_{y}}
$$

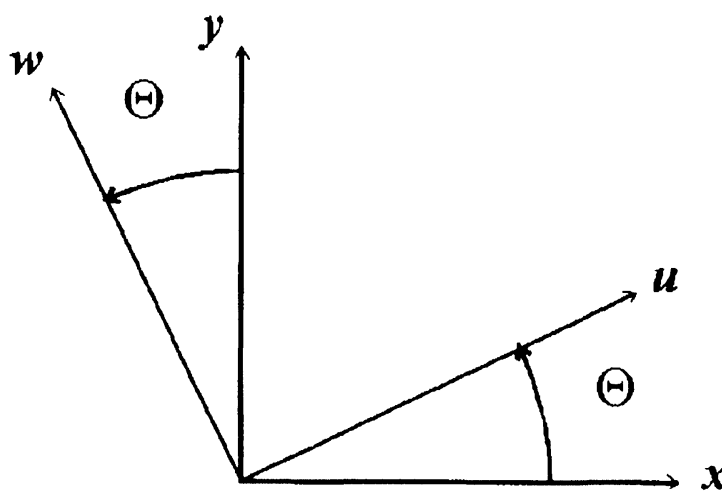

FIGURE 3 Coordinate systems.
If the rotation angle $\Theta$ corresponds to one of the main stiffness axes, and, in a particular case, is equal to either $\Theta_{1}=\arctan \phi_{1}$ or $\Theta_{2}=\arctan \phi_{2}$ where $\phi_{1}$, $\phi_{2}$ are rotor eigenfunctions (see Table I), then one coordinate ( $u$ or $w$ in the rotated system) becomes either uncoupled from the other (case (a)), or partially decoupled with minimum coupling effect (case (b), Table I). If $\Theta_{1}$ and $\Theta_{2}$ are orthogonal, which occurs in a very particular case when $\lambda_{x}+\lambda_{y}=0$, full decoupling is possible in case (a). Figure 4 presents the same data as in Figure 2, rotated by the corresponding decoupling angle calculated as $\arctan \phi_{1}$. Another decoupling angle in this case is $-68.08^{\circ}$.

The response vectors rotated the way that there is a minimum coupling effect serve better for diagnostic purposes. It is illustrated using the machine field data, following Hatch et al [1995]. Figure 5 presents gas turbine synchronous responses and Figure 6 presents the rotated data with minimum coupling.

\section{ANISOTROPIC ROTOR SYNCHRONOUS ELLIPTICAL RESPONSES SPLIT INTO FORWARD AND BACKWARD CIRCULAR ORBITS. ELLIPTICAL ORBIT MAJOR AXIS MAGNITUDE AND ANGULAR ORIENTATION}

An ellipse can be described as the locus of the sum of two constant magnitude vectors rotating with fre-

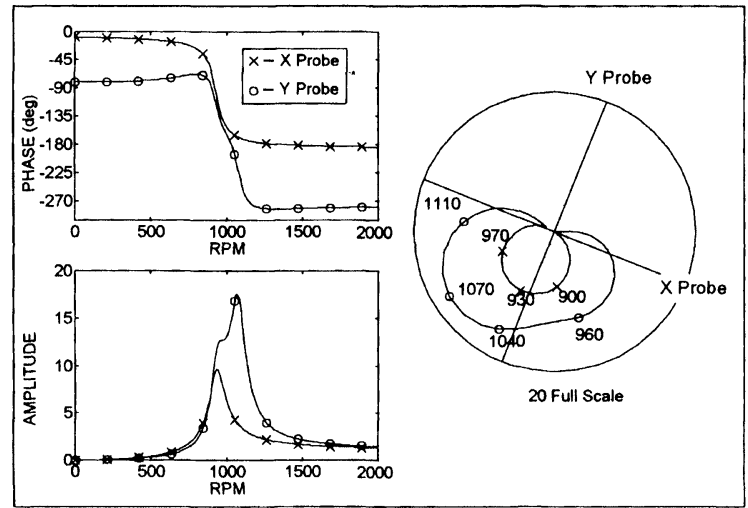

FIGURE 4 Nonsynchronous response of the rotor for $\Omega=3000$ $\mathrm{rpm}$ rotated by angle $\Theta_{1}=\arctan \phi_{1}=-21.92^{\circ}$. The same data as in Figure 2. The response marked " $X$ " is decoupled. 


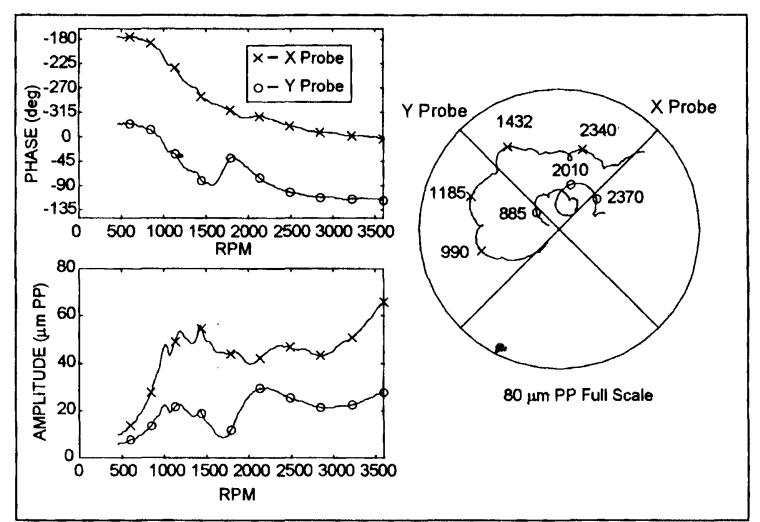

FIGURE 5 Gas turbine synchronous $(1 \times)$ response vectors in Bode and polar plot format. Displacement transducers located at $-45^{\circ},+45^{\circ}$. Phase difference at low speed is $\sim 180^{\circ}$.

quency $\omega$ in two opposite directions. A reverse method is used below to split an elliptical rotor response orbit at frequency $\omega$ into two circular orbits, one forward (in the direction of rotor rotation), and one backward (opposite to rotation). Note that if both circular orbits have the same radius (amplitude), the ellipse degenerates into a line. The larger-size orbit between the two circular ones determines whether the original elliptical orbit is forward or backward.

At a constant frequency, $\omega$ the rotor orbital response (2) can be written as follows:

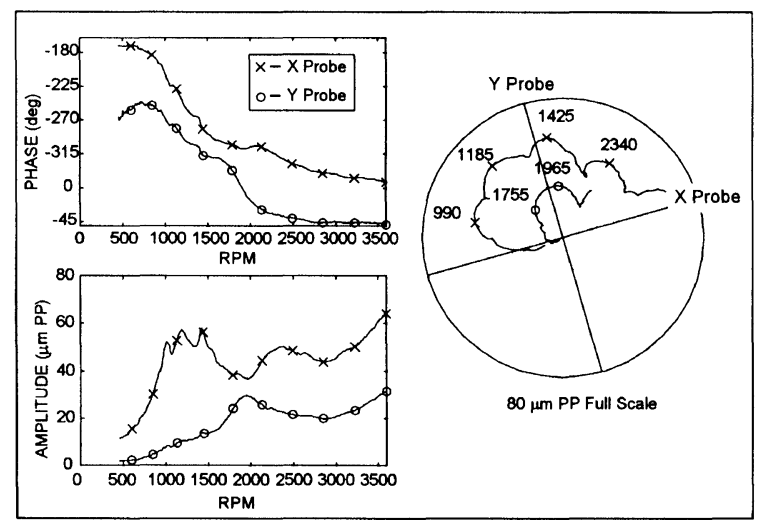

FIGURE 6 Gas turbine data from Fig. 4 rotated by $-29^{\circ}$. The "Y" response becomes partly decoupled from " $\mathrm{X}$ " response. Phase difference at low speed is $\sim 90^{\circ}$.

$$
\begin{aligned}
x+j y & =A_{x} \cos \left(\omega t+\alpha_{x}\right)+j A_{y} \cos \left(\omega t+\alpha_{y}\right) \\
& =A_{f} e^{j\left(\omega t+\alpha_{f}\right)}+A_{b} e^{j\left(-\omega t+\alpha_{b}\right)}
\end{aligned}
$$

where $A_{f}, A_{b}, \alpha_{f}, \alpha_{b}$ are amplitudes and phases of the forward and backward circular components of the orbit respectively. Using Eq. (4), they can be calculated as follows:

$$
\begin{aligned}
A_{f} & =\sqrt{A_{x}^{2}+A_{y}^{2}+2 A_{x} A_{y} \sin \left(\alpha_{x}-\alpha_{y}\right)} / 2, \\
A_{b} & =\sqrt{A_{x}^{2}+A_{y}^{2}-2 A_{x} A_{y} \sin \left(\alpha_{x}-\alpha_{y}\right)} / 2 \\
\alpha_{f} & =\arctan \frac{A_{x} \sin \alpha_{x}+A_{y} \cos \alpha_{y}}{A_{x} \cos \alpha_{x}-A_{y} \sin \alpha_{y}} \\
\alpha_{b} & =\arctan \frac{-A_{x} \sin \alpha_{x}+A_{y} \cos \alpha_{y}}{A_{x} \cos \alpha_{x}+A_{y} \sin \alpha_{y}}
\end{aligned}
$$

The amplitudes and phases of the forward and backward circular components can also be calculated directly from Eqs. (1) when the following transformation is applied: $z_{f}(t)=x+j y, z_{b}(t)=x-j y$, and then the solutions are as follows:

$$
\begin{gathered}
z_{f}=A_{f} e^{j\left(\omega t+\alpha_{f}\right)}-A_{b} e^{j\left(-\omega t+\alpha_{b}\right)}, \\
z_{b}=A_{f} e^{-j\left(\omega t+\alpha_{f}\right)}-A_{b} e^{-j\left(-\omega t+\alpha_{b}\right)}
\end{gathered}
$$

The solutions (6) for the transformed Eqs. (1) into the forward and backward mode variables $z_{f}, z_{b}$ can certainly be presented in the classical format with one amplitude and one phase for each variable. The expressions (6) emphasize the correlation between solutions for original $x, y$ and transformed variables $z_{f}$, $z_{p}$.

The backward component response amplitude and phase directly depend on anisotropic parameters, and for an isotropic system, they vanish:

$$
\begin{gathered}
A_{b}=\frac{F}{2 \Delta} \sqrt{\left(K_{x}-K_{y}\right)^{2}+\left[D\left(\lambda_{x}-\lambda_{y}\right) \Omega\right]^{2}}, \\
\alpha_{b}=-\delta+\alpha+\arctan \frac{D\left(\lambda_{x}-\lambda_{y}\right) \Omega}{K_{x}-K_{y}}
\end{gathered}
$$


The forward response amplitude and phase are as follows:

$$
\begin{gathered}
A_{f}=\frac{F}{2 \Delta} \\
\sqrt{\left(K_{x}+K_{y}-2 M \omega^{2}\right)^{2}+\left[2\left(D+D_{s}\right) \omega+D \Omega\left(\lambda_{x}+\lambda_{y}\right)\right]^{2}}, \\
\alpha_{f}=\delta-\alpha+\arctan \frac{2\left(D+D_{s}\right) \omega+D \Omega\left(\lambda_{x}+\lambda_{y}\right)}{K_{x}+K_{y}-2 M \omega^{2}}
\end{gathered}
$$

The response orbit major axis magnitude $S$ and its angular orientation $\sigma$ measured from the horizontal axis can directly be obtained as $S=A_{f}+A_{b}, \sigma=$ $0.5 \arctan \left[2 A_{x} A_{y} \cos \left(\alpha_{x}-\alpha_{v}\right) /\left(A_{x}^{2}-A_{r}^{2}\right)\right]$, or using the original parameters of the system, the latter is

$$
\sigma=\frac{1}{2} \arctan \frac{2\left(L_{y} H_{y}-L_{x} H_{y}\right)}{L_{x}^{2}-L_{y}^{2}+H_{x}^{2}-H_{y}^{2}}
$$

Figure 7 presents forward and reverse components of the synchronous response vectors of the rotor corresponding to the data illustrated in Figure 1. At low speed the forward component phase points correctly toward the unbalance angular location.

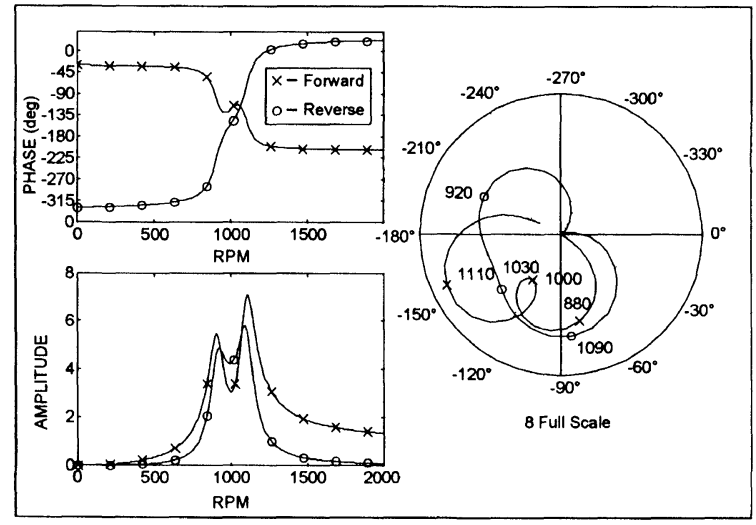

FIGURE 7 Synchronous response forward and backward components in Bode and polar plot formats. The same initial data as in Fig. 1. The backward component amplitude is larger than the forward one in the speed range 1070 to $1160 \mathrm{rpm}$. In this range the orbits are backward. Note that the low speed forward synchronous response points toward the heavy spot (here at $-30^{\circ}$ ). Amplitudes nondimensionalized by multiplying by $\mathrm{M} / \mathrm{mr}$.
Figure 8 illustrates the major axis magnitude $S$ and angular orientation $\sigma$ of the rotor synchronous orbits. Three orbits at frequencies close to resonances accompany the Bode and polar plots.

Figures 9 and 10 present the gas turbine data in the forward/reverse format, and in the format of the orbit major axis magnitude and angular orientation. Both these formats represent new tools in diagnostics of machine malfunction, and their usefulness will be established as soon as they are used.

\section{EXPERIMENTAL RESULTS DEMONSTRATING SIMULTANEOUS FORWARD AND BACKWARD ORBITING OF TWO SECTIONS OF THE ROTOR}

An experimental vertical rotor with an overhung unbalanced disk was driven through an elastic coupling by an electric motor mounted at the top. At inboard side the rotor was supported by a relatively rigid, laterally pivoting rolling element bearing. The rotor support anisotropy was achieved by sets of "horizontal," $x$, and "vertical," $y$, springs mounted to the rotor through rolling element bearings at two different axial locations. The rotor shaft was slightly bent, and also carried an unbalance. The lateral vibrations of

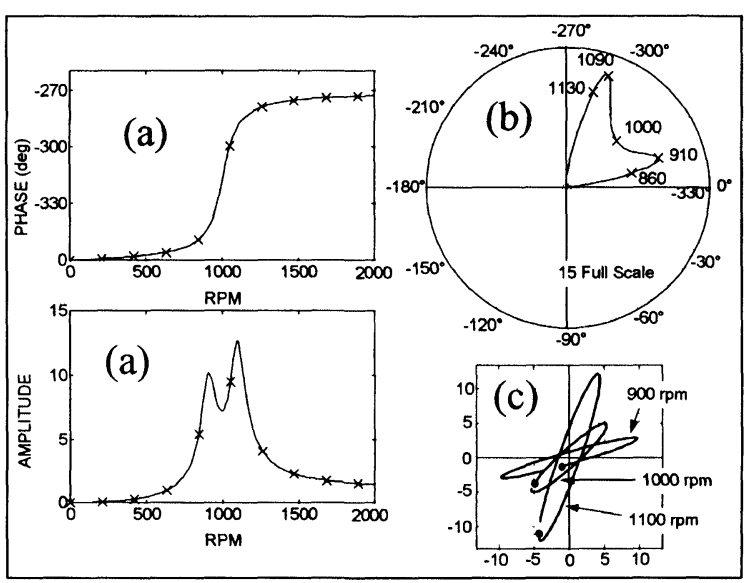

FIGURE 8 Rotor synchronous response orbit major axis magnitude and angular orientation in Bode (a) and polar plot format (b); orbits at 900,1000 , and $1100 \mathrm{rpm}(\mathrm{c})$. The same initial data as in Fig. 1. Note that the orbits at 900 and $1100 \mathrm{rpm}$ are forward and the orbit at $100(0 \mathrm{rpm}$ is backward. 


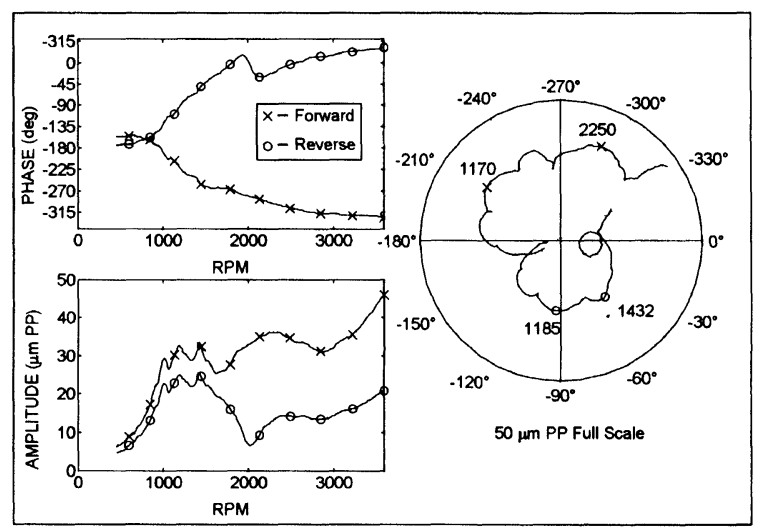

FIGURE 9 Gas turbine data from Fig. 5 in the forward and reverse $1 \times$ component Bode and polar plot format. The low speed forward response points toward the heavy spot angular orientation. Compare with Figs. 4 and 5.

the rotor were observed by three sets of $X Y$ noncontacting proximity probes (inboard, midspan, and outboard). ${ }^{1}$ The vibrational data were processed by a computerized acquisition system.

Figure 11 illustrates midspan and outboard rotor full spectrum cascades and $1 \times$ orbits at selected rotative speeds. The full spectrum contains forward and backward components of elliptical orbits at separate frequencies decomposed by Fourier transformation. The main portion of the rotor response is $1 \times$ with two distinct ("split") resonances (occurring at about $950 \mathrm{rpm}$ and $1250 \mathrm{rpm}$ ) due to the anisotropic support. Classically, between these two resonance

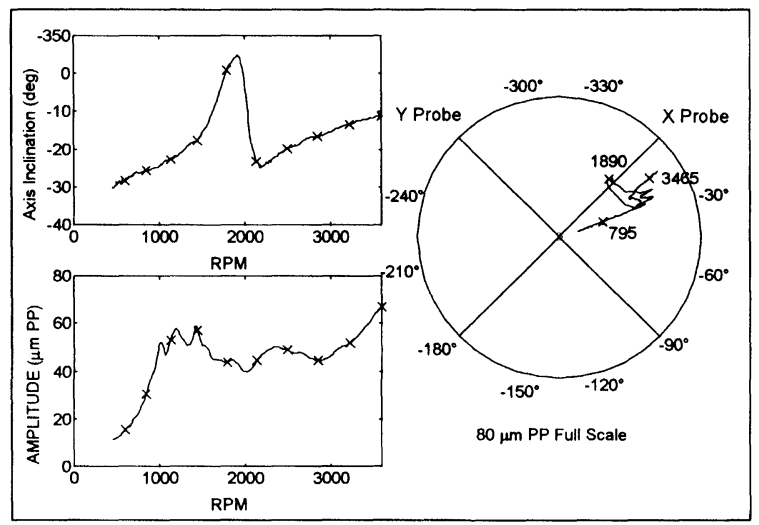

FIGURE 10 Gas turbine data from Figure 5 in $1 \times$ orbit major axis magnitude and angular orientation Bode and polar plot format. In comparison to Fig. 8, at higher speeds this data indicates a presence of the next mode. speeds, the outboard disk orbits are backward. The inboard data, which is not displayed here, looked qualitatively similar to the midspan data; this portion of the shaft responded in phase. Due to the existence of shaft bow, all $1 \times$ amplitude plots exhibited a significant response at low frequency (slow roll). The midspan bow was about twice larger than the outboard bow. The rotor average centerline plot versus rotative speed did not show any significant changes. Maximum centerline displacement was less than 4 mils, which indicated that there was no specific activity affecting the rotor centerline.

The sequence of orbits in Figure 11 reveals a phenomenon documented by the Muszynska in 1996: At certain rotative speeds the shaft midspan orbits are forward, while the outboard orbits are reverse (see the orbits at $1160 \mathrm{rpm}$ in Fig. $11^{2}$ ). This phenomenon originally raised the question: how is the shaft able to move counterclockwise at one section, and clockwise at another? Further analysis confirmed and quantified this behavioral feature. The next question concerned the deformation and stress patterns of the shaft fibers in the situation of different precession directions of two sections of the shaft. In order to assess the shaft stress, the midspan and outboard orbits at $1060 \mathrm{rpm}$ (both backward) and at $1160 \mathrm{rpm}$ were plotted again, respectively, on one figure (Figs. 12a and 13a). The numbers on the orbits corresponded to the same timing; the vectors connecting these timing points represent the outboard-to-midspan relative displacements. When drawn separately, these relative displacements reveal very similar orbits for both speeds 1060 and $1160 \mathrm{rpm}$. Both relative orbits are forward, with some amplitude differences, but very little phase change (Figs. 12b and 13b). These relative orbit graphs show that nothing unusual occurs in the shaft rotating in the 1060 to $1160 \mathrm{rpm}$ speed range. The relative orbits can be interpreted in terms of a "relative" mode, when the midspan location of the shaft is "frozen" (Fig. 14b). The full analysis of the system is presented by Muszynska [1996].

\section{FINAL REMARKS}

While anisotropy in bearing supports is often specifically incorporated in rotating machine design, as it is 

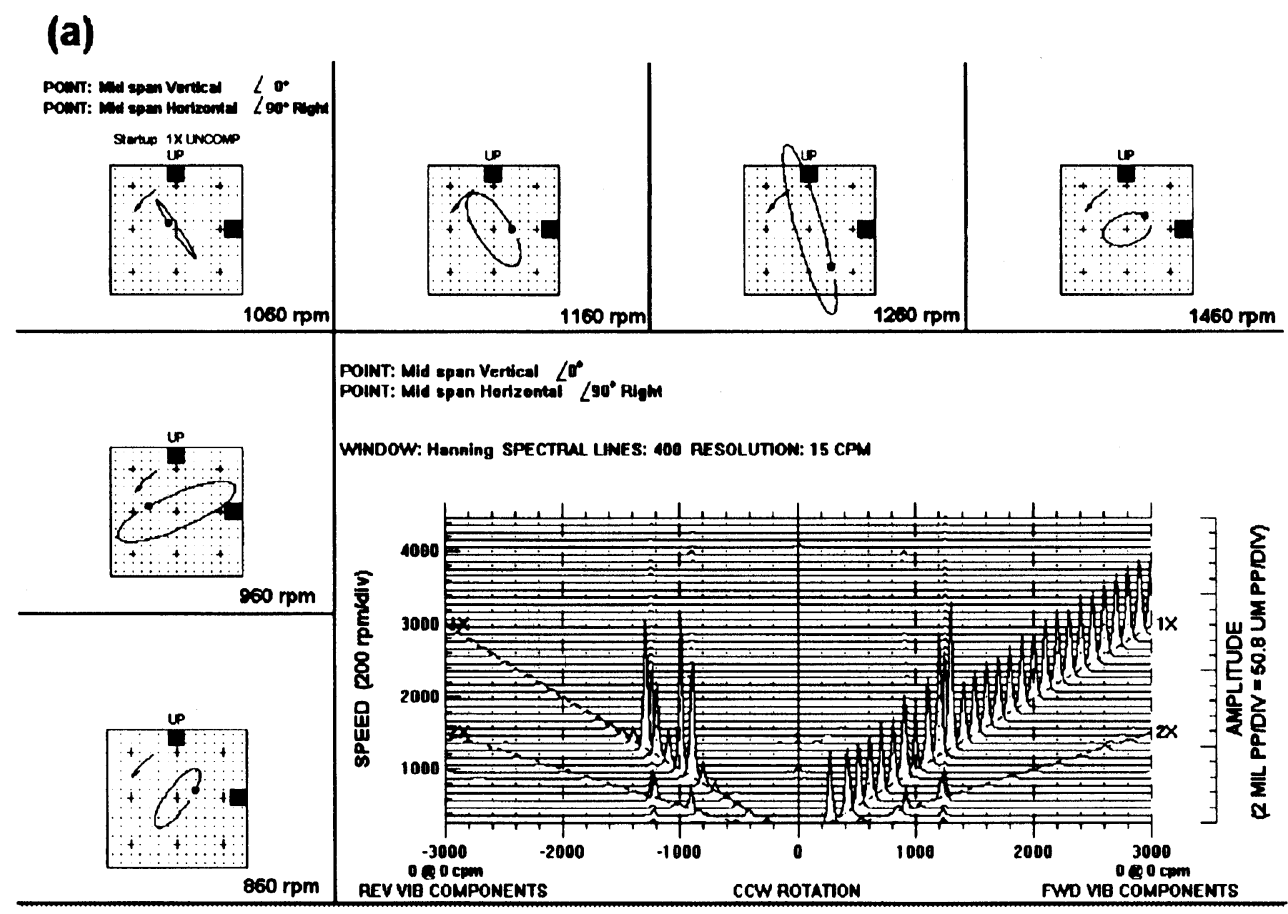

(b)

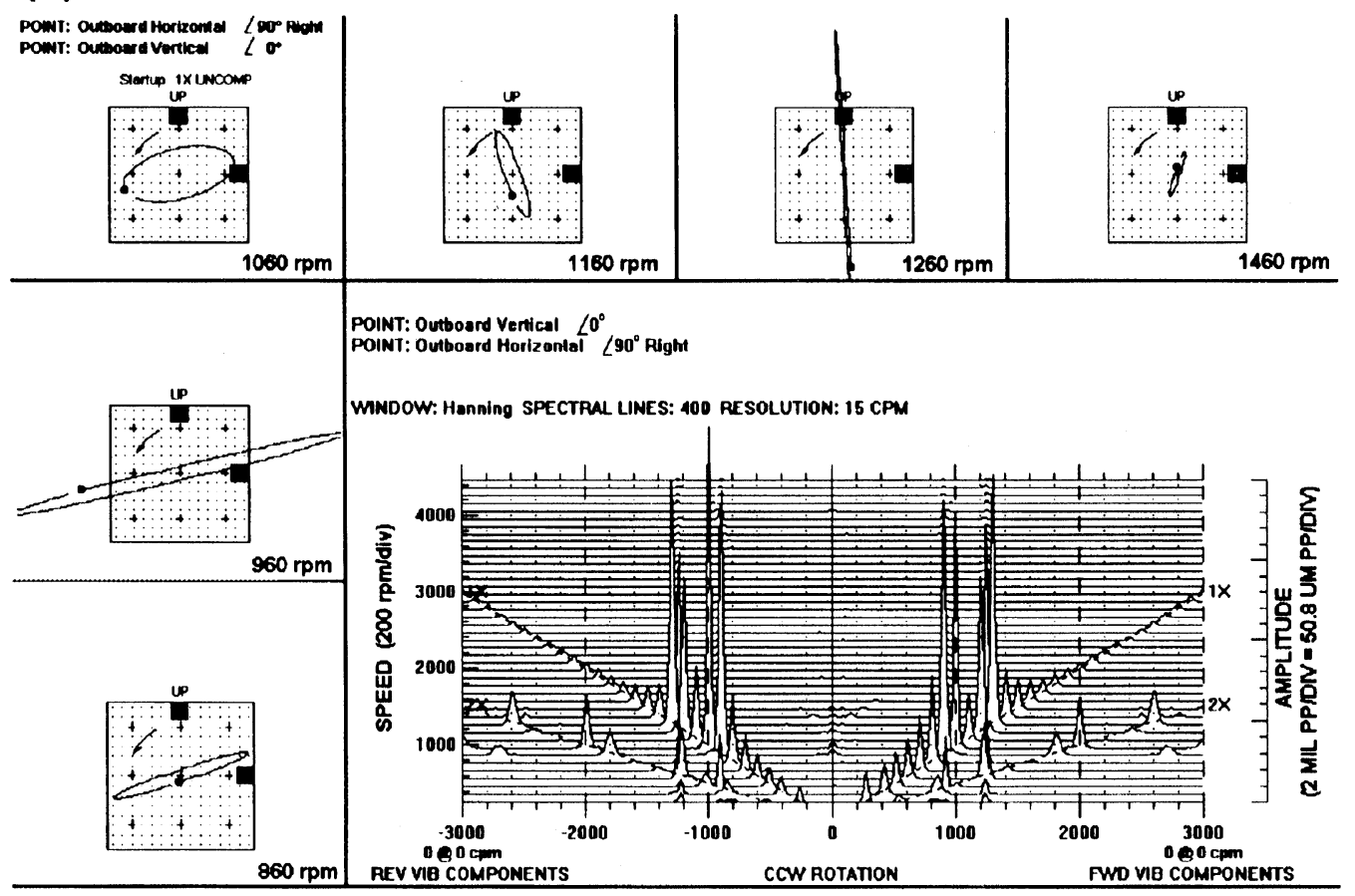

FIGURE 11 Full spectrum cascade of the rotor midspan (a) and outboard (b) "vertical" (north-south) responses accompanied by midspan and outboard filtered $1 \times$ (synchronous) orbits at selected speeds. 


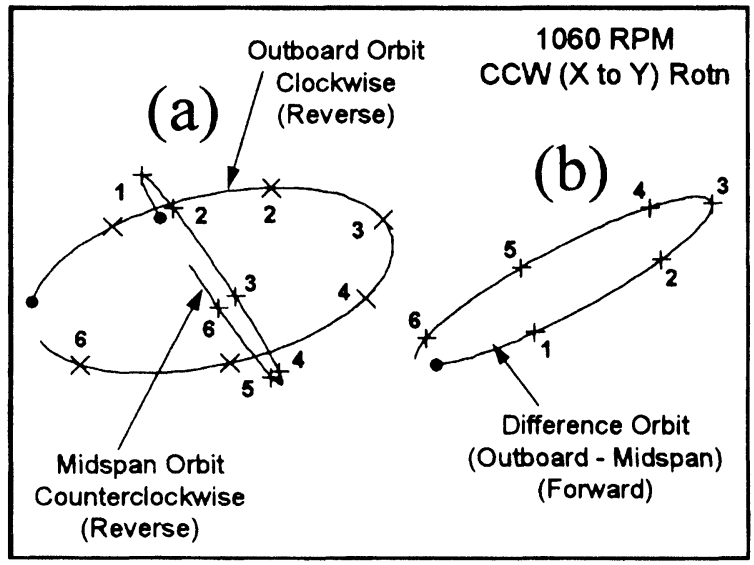

FIGURE 12 Rotor midspan and outboard orbits at $1060 \mathrm{rpm}$ from Fig. 11. Both orbits are backward. Differential orbit (b) is forward.

known to enhance the stability of the rotor (see, for instance. Handbook of Rotordynamics, 1993), it also introduces, in some rotative speed ranges, the inevitable, unbalance-related, backward precessional motion, resulting in damaging rotor reversal stresses. The brighter side of this is related to the fact that rotative speed bands where the backward precession takes place are relatively narrow, and they occur near closely spaced rotor natural frequencies which are normally avoided as operational speeds. The danger,

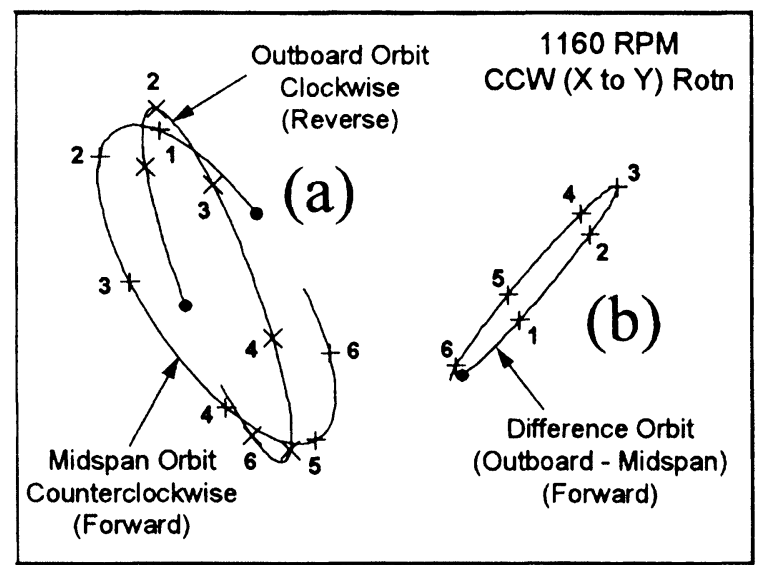

FIGURE 13 (a) Rotor midspan and outboard orbits at $1160 \mathrm{rpm}$ The midspan orbit is forward. the outboard orbit is backward. (b) Differential orbit is again forward with no phase change compared to Fig. 12. (a) Original model

(b) Differential mode
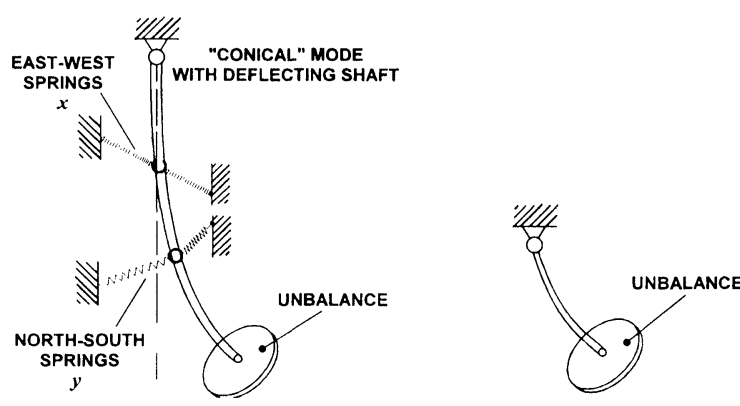

FIGURE 14 (a) Rotor original vibration mode. (b) Rotor relative vibration mode with "frozen" midspan location.

however, always exists during startups and shutdowns of the machine. Careful balancing and shaft straightening considerably improve rotor transient condition operations. Also, as shown by Corley [1986], a sufficient amount of damping in the rotor/ support system may effectively suppress the backward precessional motion.

It has been shown in the paper that vibration data processing using filtered forward/backward orbit components and orbit major axis amplitude and its orientation may be useful for machine malfunction diagnostics purposes. As new tools, the usefulness of these new formats will be proven following accumulated experience when specific machine malfunctions will be associated with a growth (or decrease) of a specific response component.

\section{NOMENCLATURE}

$A_{f}, A_{l}, \alpha_{f}, \alpha_{l}$ Amplitudes and phases of forward and backward circular components of rotor elliptical orbits respectively

$A_{r}, A_{v}, \alpha_{v}, \alpha_{v}$ Rotor response amplitudes and phases in $x$ and $y$ direction respectively

$A_{u}, A_{u}, \alpha_{u}, \alpha_{11}$ Rotor response amplitudes and phases in $u$ and $w$ direction respectively 
$D_{s}, M$

$F, \omega, \delta$

$K_{x}, K_{y}$

respectively

Bearing or seal fluid radial damping coefficient

Rotor modal damping and mass respectively

External exciting nonsynchronously rotating force amplitude, frequency, and angular orientation

Rotor/supporting structure stiffnesses in $x$ and $y$ directions

$s_{1}, v=1, \ldots, 4$ Eigenvalues

$S, \sigma \quad$ Orbit major axis magnitude and its angular orientation

$t \quad$ Time

$u(t), w(t) \quad$ Rotor lateral deflections in coordinates rotated by a constant angle $\Theta$

$x(t), y(t) \quad$ Rotor lateral deflections in two orthogonal directions

$z_{f}(t), z_{1}(t) \quad$ Rotor forward and backward mode variables

$\lambda_{x}, \lambda_{1}$ Fluid circumferential average velocity ratios

$\Theta$ Coordinate or transducer rotation angle

$\phi_{1}, \phi_{2}$

$\Omega$ Eigenfunctions

Rotative speed

\section{References}

Benty Nevada Mechanical Diagnostic Service Reports. 1989-1994
Corley, J. E.. 1986. The Importance of Being Stiff: The Effects of Pedestal Stiffness on Rotor Dynamics, Vibration Institute 10th Annual Meeting Proceedings, Las Vegas, Nevada.

Gunter, E. J., Fang. Z.. 1993. Forward and Backward Critical Speeds and Forced Response of an Overhung Rotor With Asymmetrical Bearing Supports. University of Virginia. School of Engineering \& Applied Science, Department of Mechanical \& Aerospace Engineering.

Handbook of Rotordinamics, 1993. Ed. F. F. Ehrich. McGraw Hill, Inc., New York, Auckland, Bogota, Montreal, New Delhi.

Hatch, C. T., Bently, D. E., 1995. Anisotropic Rotor Response and Probe Data Transformation to Improve Machinery Diagnostic Capability," BRDRC Report No. 1/95.

Muszynska. A., 1988. Improvements in Lightly Loaded Rotor/ Bearing and Rotor/Seal Models, Trans. of the ASME, Journal of Vibration, Acoustics, Stress and Reliability in Design, v. 110, No. 2.

Muszynska, A., 1989. Two-Mode Rotor With Anisotropic Supports and Nonsymmetric Tangential Force, BRDRC Report No. 2/89.

Muszynska, A., 1994. Application of Multi-Mode Modal Models in Rotor Dynamics, Proceedings of ISROMAC-5, Hawaii.

Muszynska, A., 1994. Forward and Backward Precession of a Vertical Anisotropically Supported Rotor, Journal of Sound and Vibration, Vol. 192.

Perez, G., Private communication.

Vance, J. M., 1993. Rotordynamics of Turbomachinery, John Wiley \& Sons, New York, Brisbane, Toronto, Singapore.

Xia, S., Wu, X., Wang. G., 1987. An Investigation of Vibration Characteristics of Rotor and Rotor Support System for Locomotive Gas Turbine, Rotating Machinery Dynamics, ASME. Boston.

\section{Endnotes}

1. Each of the two orthogonally oriented proximity probes are actually located in horizontal planes. They measure the vertical rotor lateral vibrations in two horizontal directions. Following, however, the oscilloscope convention, these two lateral directions are called here "vertical" and "horizontal."

2. The direction of precession (orbiting) is indicated by the sequence blank/bright (blank/dot) on the orbit.) 


\section{ait \\ ENERGY MATERIALS}

M A N E Y publishing

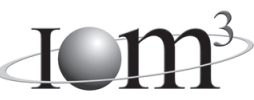

\section{Materials Science \& Engineering for Energy Systems}

Maney Publishing on behalf of the Institute of Materials, Minerals and Mining

The Institute of Materials, Minerals \& Mining

Economic and environmental factors are creating ever greater pressures for the efficient generation, transmission and use of energy. Materials developments are crucial to progress in all these areas: to innovation in design; to extending lifetime and maintenance intervals; and to successful operation in more demanding environments. Drawing together the broad community with interests in these areas, Energy Materials addresses materials needs in future energy generation, transmission, utilisation, conservation and storage. The journal covers thermal generation and gas turbines; renewable power (wind, wave, tidal, hydro, solar and geothermal); fuel cells (low and high temperature); materials issues relevant to biomass and biotechnology; nuclear power generation (fission and fusion); hydrogen generation and storage in the context of the 'hydrogen economy'; and the transmission and storage of the energy produced.

As well as publishing high-quality peer-reviewed research, Energy Materials promotes discussion of issues common to all sectors, through commissioned reviews and commentaries. The journal includes coverage of energy economics and policy, and broader social issues, since the political and legislative context influence research and investment decisions.

\section{CALL FOR PAPERS}

Contributions to the journal should be submitted online at http://ema.edmgr.com

To view the Notes for Contributors please visit: www.maney.co.uk/journals/notes/ema

Upon publication in 2006, this journal will be available via the Ingenta Connect journals service. To view free sample content online visit: www.ingentaconnect.com/content/maney

For further information please contact:

Maney Publishing UK

Tel: +44 (0)113 2497481 Fax: +44 (0)1132486983 Email: subscriptions@maney.co.uk

or

Maney Publishing North America

Tel (toll free): 8662975154 Fax: 6173546875 Email: maney@maneyusa.com

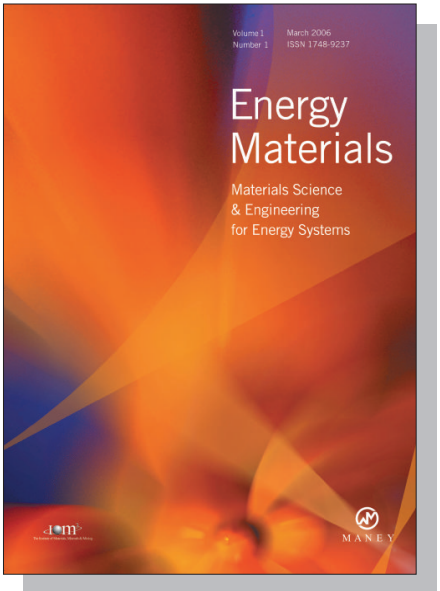

EDITORS

Dr Fujio Abe

NIMS, Japan

Dr John Hald, IPL-MPT, Technical University of Denmark, Denmark

Dr R Viswanathan, EPRI, USA

\section{SUBSCRIPTION INFORMATION}

Volume 1 (2006), 4 issues per year

Print ISSN: 1748-9237 Online ISSN: 1748-9245

Individual rate: $£ 76.00 / U S \$ 141.00$

Institutional rate: $£ 235.00 /$ US $\$ 435.00$

Online-only institutional rate: $£ 199.00 / U S \$ 367.00$

For special $\mathrm{IOM}^{3}$ member rates please email

subscriptions@maney.co.uk

\section{For further information or to subscribe online please visit www.maney.co.uk}



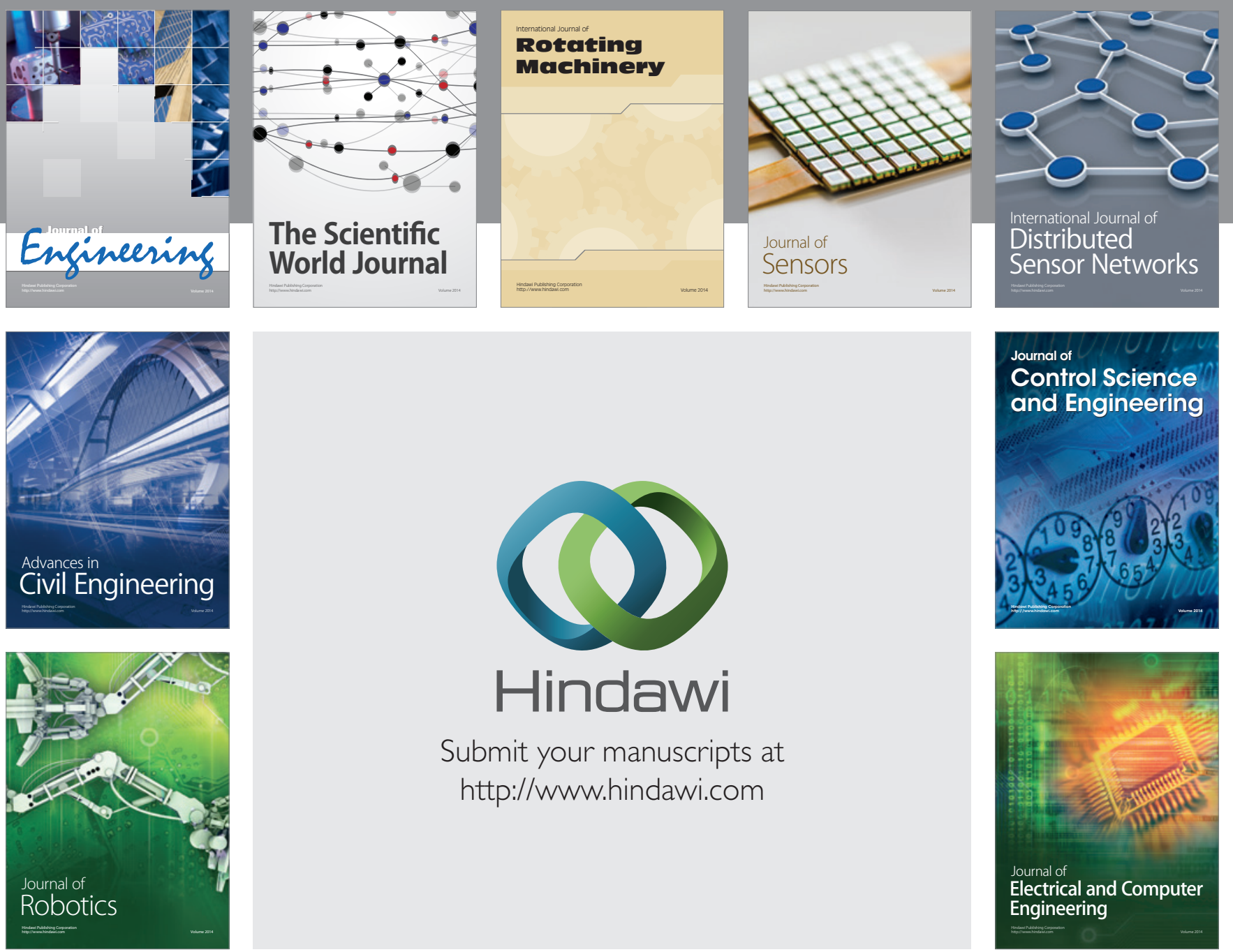

Submit your manuscripts at

http://www.hindawi.com
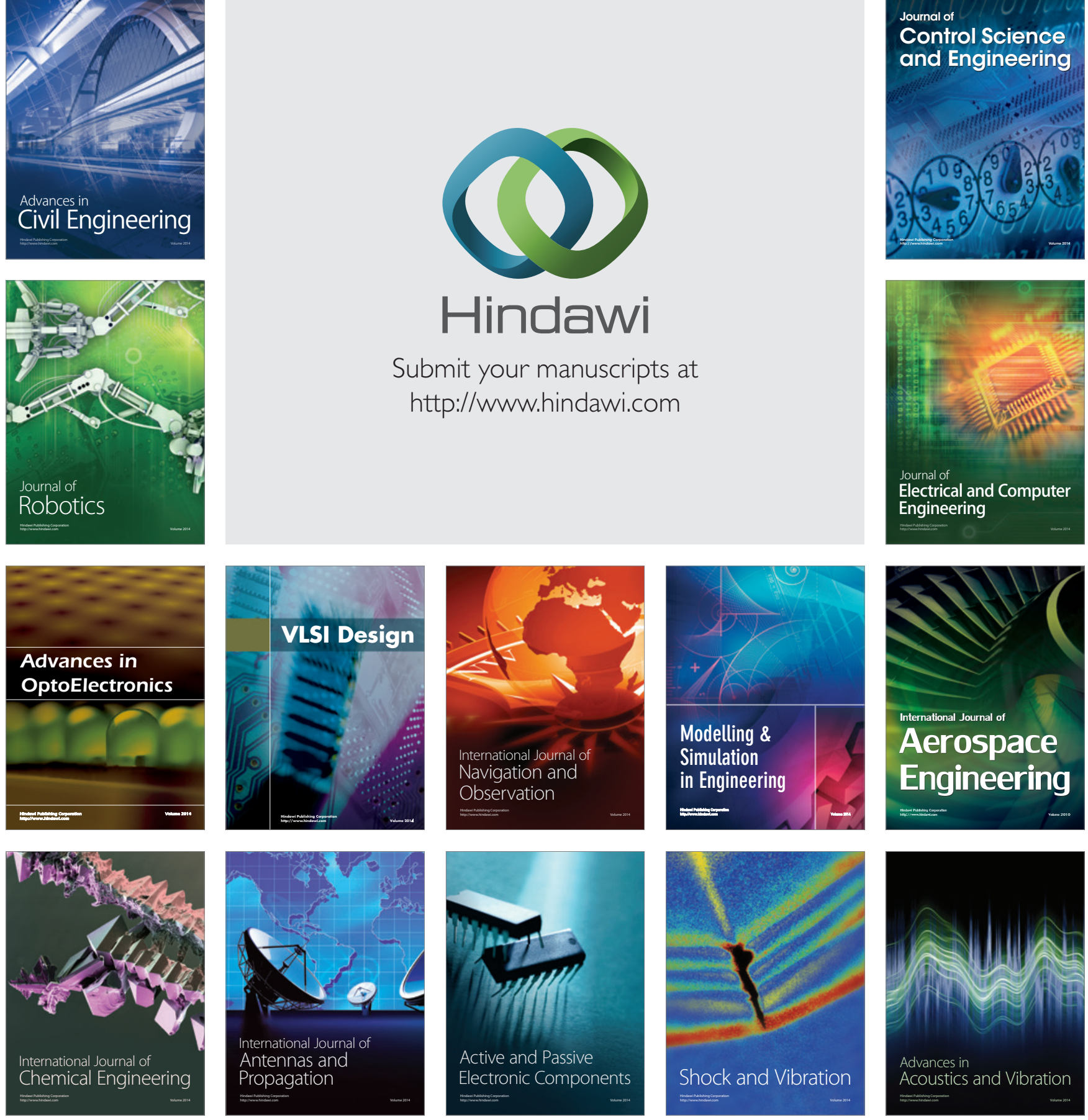